\title{
The protective effect of Epimedii Folium and Curculiginis Rhizoma on Alzheimer's disease by the inhibitions of NF-KB / MAPK pathway and NLRP3 inflammasome
}

\author{
Zhou Lan ${ }^{1}$, Guangjing $\mathrm{Xie}^{2}$, Meng Wei ${ }^{1}$, Ping Wang ${ }^{2}$ and Lvyi Chen ${ }^{3}$ \\ ${ }^{1}$ School of Pharmacy, Hubei University of Chinese Medicine, Wuhan, P. R. China \\ ${ }^{2}$ School of Basic Medicine, Hubei University of Chinese Medicine, Wuhan, P. R. China \\ ${ }^{3}$ School of Pharmaceutical Sciences, South-Central University for Nationalities, Wuhan, P. R. China \\ Correspondence to: Lvyi Chen, email: clyhappy05@163.com \\ Ping Wang, email: pwang54@aliyun.com
}

Keywords: the water extracts of Epimedii Folium and Curculiginis Rhizoma (EX), Alzheimer's disease, amyloid $\beta$, neuroinflammation, Gerotarget

Received: August 23, $2016 \quad$ Accepted: October 05, $2016 \quad$ Published: May 23, 2017

Copyright: Lan et al. This is an open-access article distributed under the terms of the Creative Commons Attribution License 3.0 (CC BY 3.0 ), which permits unrestricted use, distribution, and reproduction in any medium, provided the original author and source are credited.

\section{ABSTRACT}

The purpose of the current study was to explore the effects of the water extracts of Epimedii Folium and Curculiginis Rhizoma (EX) on A $\beta$-induced Alzheimer's disease. $A \beta_{1-42}$ was stereotaxically injected bilaterally into the dorsal hippocampus, and then the rats were orally received EX at the doses of $2 \mathrm{~g} / \mathrm{kg}$ and $6 \mathrm{~g} / \mathrm{kg}$ for 30 days. Behavior was monitored through Morris water maze test. The neuroprotective effect of EX were examined with methods of histochemistry and biochemistry. EX reduced the contents of pro-inflammatory cytokines tumor necrosis factor-alpha (TNF-a), interleukin-1 beta (IL-1 $\beta$ ) and interleukin-6 (IL-6) in hippocampus and cortex. EX also reduced the levels of malondialdehyde (MDA) and increased superoxide dismutase (SOD), catalase (CAT), glutathione (GSH) and glutathione peroxidase (GSH-PX) in the serum. Immunohistochemical analysis demonstrated that EX inhibited the expressions of NLRP3. In addition, we further confirmed that EX suppressed the expression of the NLRP3 inflammasome. EX inhibited the phosphorylations MAPKs, nuclear factor $K B$ (NF-KB), myeloid differentiation factor 88(MyD88), cathepsin B. In conclusion, these results suggest that EX may be a potential agent for treating Alzheimer's disease.

\section{INTRODUCTION}

Alzheimer's disease (AD), a common form of dementia, is a common neurogenerative disorder characterized by extracellular deposition of A beta $(\mathrm{A} \beta)$ plaques and intracellular accumulation of hyperphosphorylated tau protein and NFTs (neurofibrillary tangles) [1]. The progressive accumulation of $A \beta$ into oligomers and plaques and the inter-neuronal NFTs contribute to synaptic loss, neuronal dysfunction and death within vulnerable brain regions, leading to the progressive loss of memory and executive functions [2]. Currently, there are more than 38 million $\mathrm{AD}$ patients in the worldwide and every four seconds a new patient is diagnosed with $\mathrm{AD}$. This number is expected to double every 20 years and to reach 115 million in 2050 due to the increasing aging population [3]. By the end of 2014, the number of Chinese aged 60 or older reached 212 million. The number of aging people is predicted to swell to more than one-third of the population by 2050, when China will become one of the countries most heavily burdened by $\mathrm{AD}$ [4]. Therefore, the effective treatment of $\mathrm{AD}$ becomes more urgent and remains a challenge.

At present, it is well accepted that $\mathrm{AD}$ is marked by neuroinflammatory events mediated by the resident neuroimmune cells of the brain, involving microglia and astrocytes [5]. One of the key pro-inflammatory cytokines involved in the neuroinflammatory process is interleukin$1 \beta$ (IL-1 $\beta$ ) [6]. IL-1 $\beta$ released from the plaque-surrounding microglia after stimulation with $A \beta$ may contribute to deficits in long-term memory and neuronal dysfunction by promoting the formation of dystrophic neurite and 
its direct neurotoxicity [7]. The production and secretion of IL-1 $\beta$ require signals mediated by Toll-like receptors (TLRs) and NOD-like receptors (NLRs) [8]. On one hand, TLRs leads to the transcriptional induction of the pro-IL$1 \beta$ via the activation of nuclear factor- $\kappa \mathrm{B}(\mathrm{NF}-\kappa \mathrm{B})$ [9]. On the other hand, $A \beta$ can active the NLRP3 inflammasome, a complex containing the apoptosis-associated specklike protein (ASC), the protease caspase-1 and NLRP3, in microglia, which is fundamental for the secretion of IL- $1 \beta$. IL- $1 \beta$ is primarily produced by pro-IL- $1 \beta$ to become biologically active. This process is mediated by the NLRP3 inflammasome which induces the activation of pro-caspase- 1 to promote the maturation of pro-IL-1 $\beta$ [10]. These findings suggest that inflammation is proposed as a vital effector of AD.

To date, the anti-AD drugs mainly include acetylcholinesterase inhibitors, such as donepezil, rivastigmine and galantamine, and $\mathrm{N}$-methylD-aspartic acid (NMDA) glutamate receptor antagonists (memantine) [11]. These therapeutic interventions for $\mathrm{AD}$, however, have been primarily limited to treating symptoms without the ability to target the protection of the neurons. Once the intervention is interrupted, the symptoms of $\mathrm{AD}$ will be recovered. Consequently, there has been a great demand for new anti-AD agents capable of acting on multiple cytokines or mediators of inflammation. Fortunately, the effective treatments for $\mathrm{AD}$ are available from practitioners of traditional Chinese medicine. Traditional Chinese medicines exert their pharmacological effects through a multi-component and multi-target way in addition to their fewer side-effects, which provide the advantages and wide application possibilities compared with the pure drug with limited efficacy and some side effects. Therefore, researches have aimed at developing potent AD's drugs from Chinese medicines.

Epimedii Folium is the dried leaf of Epimedium brevicornu Maxim. and other 3 species in the genus Epimedium of the family Berberidaceae. It is warm in nature and acrid and sweet in taste. It can replenish Shen-yang, strong bones and muscles, and dispel wind and dampness. It has been commonly used for the treatment of atherosclerosis and neuropathy in traditional Chinese medicine. Moreover, Epimedii Folium has been proven to possess a wide range of efficacy including anti-inflammatory, anti-oxidant, anti-tumor and neuroprotective effects, etc. Pharmacological studies have also showed that icariin, a natural flavonoid compound extracted from Epimedii Folium, can remarkably attenuate cognitive deficits in several different AD's models [12-13].

Curculiginis Rhizoma is the dried rhizoma of Curculigo orchioides Gaertn. (It belongs to the family Amarylidaceae). Curculigo orchioides Gaertn. is a small herb widely distributed in China, Malaya, Japan, India and Australia. It has the properties of invigorating Shen-yang, expelling cold and eliminating dampness. It has been commonly used to treat impotence, cold sperm and aging diseases as a traditional Chinese medicine in China for a long time. In addition, recent studies have manifested that compounds isolated from Curculiginis Rhizoma had a wide spectrum of pharmacological activities, including neuro-protective, anti-oxidant, anti-inflammatory, antibacterial, anti-osteoporotic and estrogenic effects, etc [14$15]$.

In China, Epimedii Folium and Curculiginis Rhizoma are often used together as herb pairs in formula on clinic in order to obtain a synergistic effect for reinforcing Shen-yang and relieving pain to treat aging diseases. For example, the famous traditional Chinese medicine formula Er-Xian decoction containing Epimedii Folium and Curculiginis Rhizoma as the main herbs has been used for the treatment of osteoporosis disorders, menopausal syndrome and aging diseases for several decades [16]. In this formula, Epimedii Folium and Curculiginis Rhizoma are succinctly combined with the therapeutic merit of warming Shen-yang. Although Epimedii Folium and Curculiginis Rhizoma are excellently combined as herb pair on clinic, the evaluation of effects and mechanisms of this herb pair are lack. A number of researches have more focused on the pharmacological activities of Epimedii Folium and Curculiginis Rhizoma used alone other than together. Hence, the mechanisms of the combined extracts of Epimedii Folium and Curculiginis Rhizoma were investigated in $\mathrm{A} \beta_{1-42}$-induced AD rats in this study.

\section{RESULTS}

\section{TOF-MS analysis of EX}

The typical TOF-MS chromatogram of EX was shown in Figure 1. By comparing both the retention times of the reference standards and the spectra of MS, six compounds, including Orcinol glucosid (1), Curculigoside (2), Epimedin A (3), Epimedin B (4), Icariin (5), Baohuoside I (6) were well indentified.

\section{Effect of EX on spatial learning and memory ability}

The spatial learning and memory ability of animals was assessed by Morris water maze test. Figure 2 showed that the mean latency to find the platform declined progressively during the five training days. The $A \beta_{1-42}{ }^{-}$ treated rats remarkably spent longer time on finding the platform than the control ones did on the second day onwards $(p<0.05)$. These results revealed that the $\mathrm{A} \beta_{1-42^{-}}$ treated rats had obvious cognitive impairment. Moreover, the increase of escape latency was shortened respectively by EX $2 \mathrm{~g} / \mathrm{kg}$ from the third to fourth days $(p<0.01 \mathrm{vs}$. the $\mathrm{A} \beta_{1-42}$-treated model rats) and by EX $6 \mathrm{~g} / \mathrm{kg}$ from the first 
to fifth days ( $p<0.05 v s . \mathrm{A} \beta_{1-42}$-treated model rats). Figure 2 illustrated the swim paths of rats in the second trial on the second and the fifth days in this test. Rats tended to explore all four quadrants of the pool in the second day. On the fifth day, the control rats swam in the direction of the platform, however, $\mathrm{A} \beta_{1-42}$-treated rats took longer swimming paths. In the probe test (Figure 2), the control rats spent almost twice more of the time in the quadrant where the platform was once placed than the $A \beta_{1-42}$-treated model rats $(p<0.05)$. Besides, compared with the $\mathrm{A} \beta_{1 \text {. }}$ ${ }_{42}$-treated model rats, EX ( 2 and $6 \mathrm{~g} / \mathrm{kg}$ ) treated rats took longer time in the target quadrant ( $p<0.05$, respectively).

\section{Effects of EX on pro-inflammatory cytokines (IL- $1 \beta$, IL-6 and TNF- $\alpha$ )}

To assess the impact of EX on the $\mathrm{A} \beta_{1-42}$-mediated inflammatory response, the ELISA kits were applied to determine the production of IL-1 $\beta$, IL- 6 and TNF- $\alpha$. As shown in Figure 3-4, the elevated levels of IL-1 $\beta$, IL-6 and TNF- $\alpha$ significantly were found in the mice which were subjected to intrahippocampal $A \beta_{1-42}$ injection. However, EX treatments $(2 \mathrm{~g} / \mathrm{kg}, 6 \mathrm{~g} / \mathrm{kg})$ significantly declined the production of IL-1 $\beta$, IL- 6 and TNF- $\alpha$ in the hippocampus and cortex after intrahippocampal $\mathrm{A} \beta_{1-42}$ injection. These results demonstrated that EX might ameliorate $A \beta_{1-42}$ induced overproduction of pro-inflammatory cytokines, which was evidenced by the reverse effect of EX on the increase in the levels of IL-1 $\beta$, IL- 6 and TNF- $\alpha$.

\section{Effects of EX on oxidation-related indicators (SOD, MDA, GSH-Px and CAT)}

To examine the anti-oxidative property of EX, the levels of MDA, SOD, CAT and GSH-Px in serum were detected. As depicted in Figure 5, A $\beta_{1-42}$ injection was proved to significantly decline the SOD, CAT and GSH$\mathrm{Px}$ activities with the increase of MDA content in the serum. On the contrary, treatment with $\mathrm{EX}(2,6 \mathrm{~g} / \mathrm{kg})$ remarkably restored the activities of SOD, CAT and GSHPx to different degree in the serum compared with those in model group. Additionally, an elevation was exhibited in the MDA content after EX $(2,6 \mathrm{~g} / \mathrm{kg})$ treatment with a more apparent increase of MDA at the dose of EX (6 g/ $\mathrm{kg}$ ), as compared with the model group of merely $\mathrm{A} \beta_{1-42}$ injection.

\section{Effects of EX on NLRP3 inflammasome activation}

Mounting evidence had highlighted that NLRP3 activation which was related to $A \beta$ degradation played an essential role in the pathological progression of AD [17]. Immunohistochemical staining showed that EX could attenuated the expressions of NLRP3 in brains (Figure 6) . Accordingly, we investigated the effect of EX on NLRP3 inflammasome activation. The data depicted in Figure 7 illustrated the highly expressed NLRP3, ASC, Caspase-1, and IL-1 $\beta$ in model group, while the administration of EX $(2 \mathrm{~g} / \mathrm{kg}, 6 \mathrm{~g} / \mathrm{kg}$ ) and dramatically inhibited the expressions of them to varying degrees with GAPDH. Results obtained from western blots (Figure 8) suggested the increased expression of MAPK- NF- $\kappa \mathrm{B}$ axis in model rats and decreased of them in $\mathrm{EX}(2 \mathrm{~g} / \mathrm{kg}, 6 \mathrm{~g} / \mathrm{kg})$ treated groups, which further confirmed the involvement of inflammation pathway in $A \beta_{1-42}$-induced $A D$.

\section{DISCUSSION}

In the present study, we investigated the effect of EX on cognitive function and neuroinflammatory in $\mathrm{A} \beta$-treated mice. $\mathrm{A} \beta_{1-42}$ is reported to be generated by

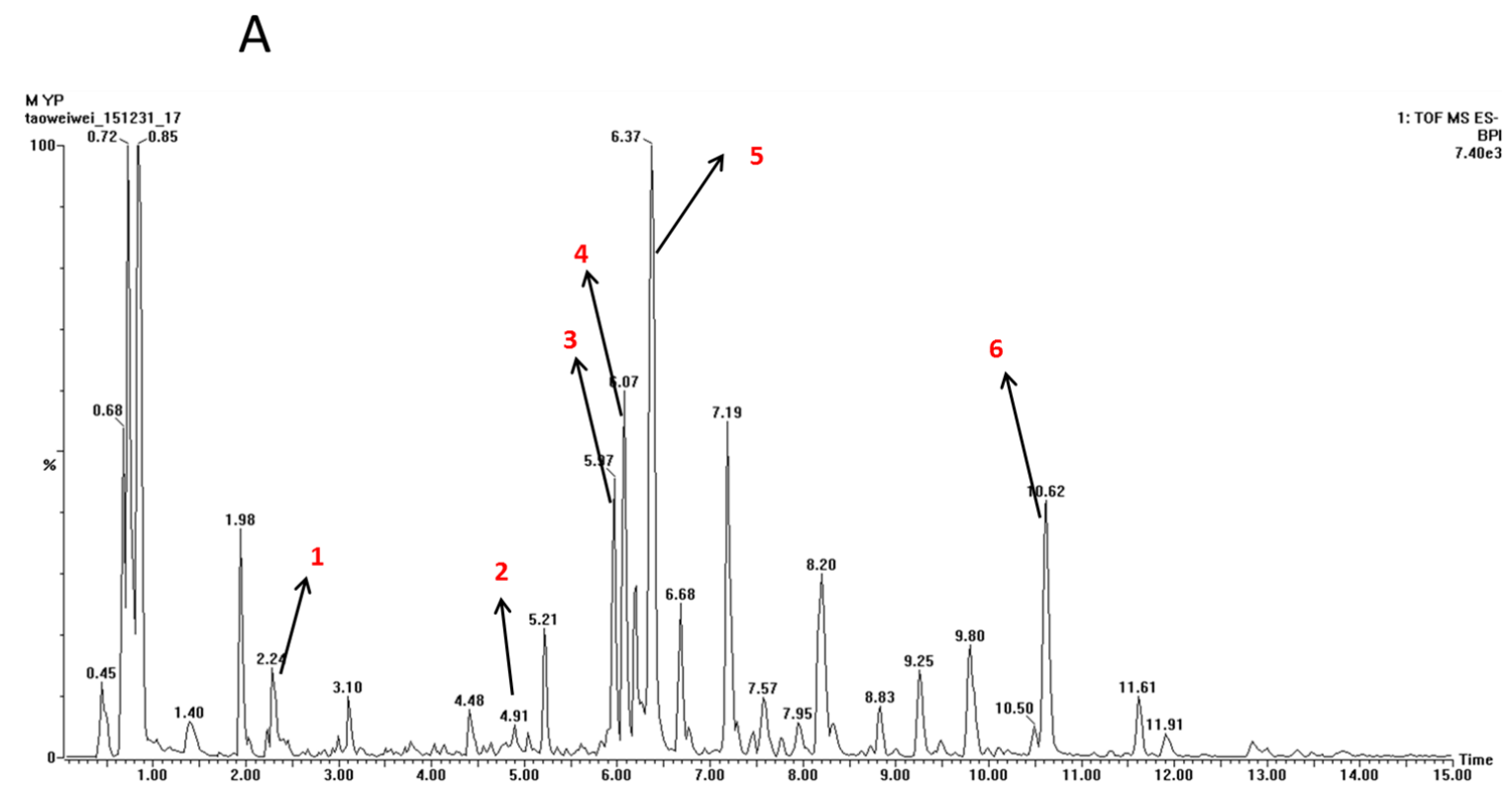



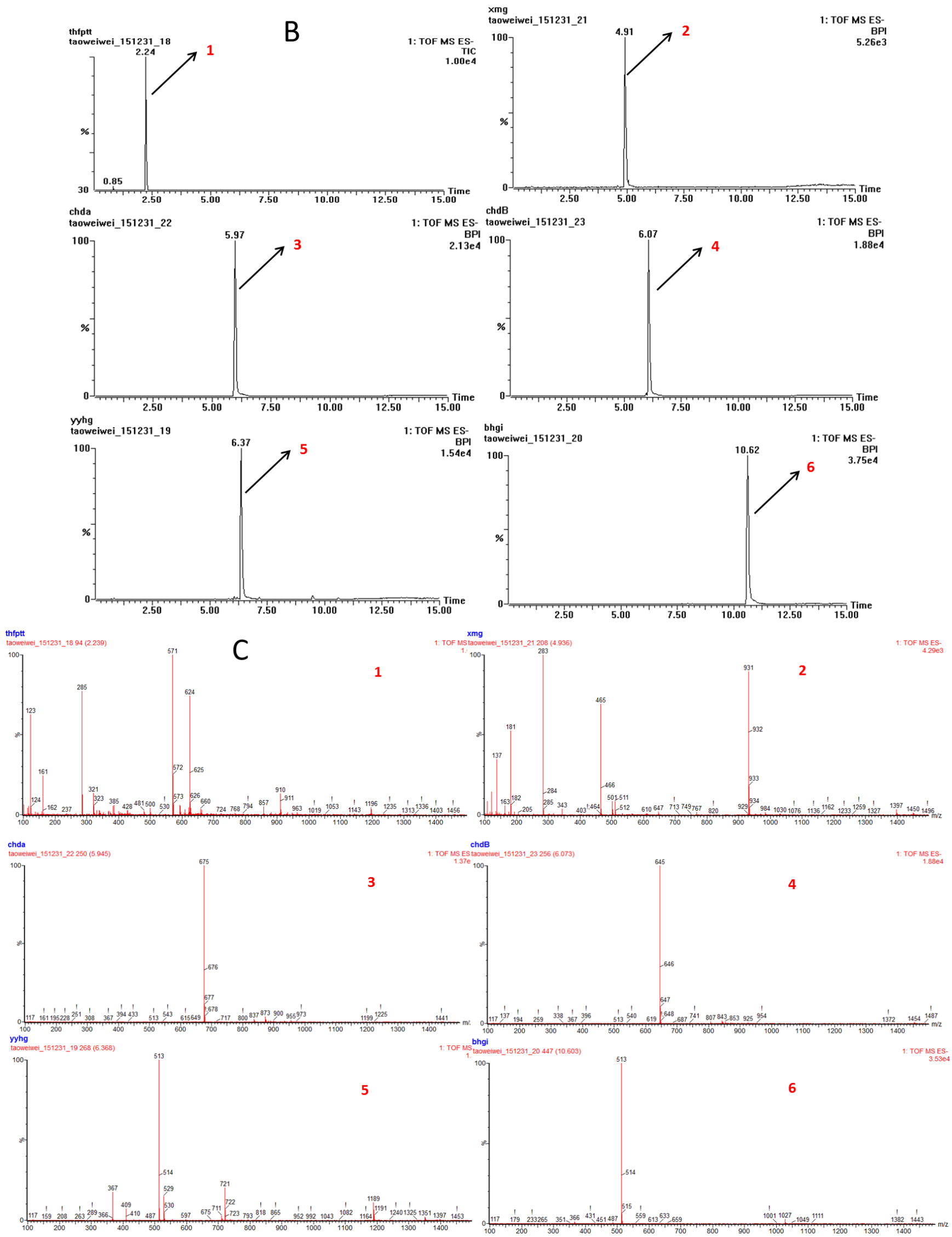

Figure 1: TOF-MS chromatogram of EX. (1) Orcinol glucosid; (2) Curculigoside; (3) Epimedin A; (4) Epimedin B; (5) Icariin; (6) Baohuoside I. 
successive cleavage of the amyloid precursor protein (APP) through $\beta$-secretase and $\gamma$-secretase [18], which was employed to induce AD to verify the effects of EX . And the AD model was successfully developed by the assessment of Morris water maze test (MWM), which was in line with the findings from prior works [19]. On the one hand, poor performance was observed in the MWM test in $\mathrm{A} \beta_{1-42}$-induced cognitive dysfunction, which was in agreement with previous literatures [20]. On the other hand, EX considerably declined the escape latency of $\mathrm{AD}$ rats in the MWM test, implying that EX could enhance hippocampal-mediated learning and memory abilities in AD rats.

Previous research has demonstrated that inflammation plays a vital role in the progression of poor cognitive performance [21]. It was suggested that inflammation was one of the mechanisms related to AD characterized by progressive memory disorders and cognitive dysfunction [22]. The released cytokines, in particular IL-1 $\beta$, IL- 6 and TNF- $\alpha$, are the major effectors of the neuroinflammatory signals, and can affect neurophysiologic mechanisms regarding cognition and memory [23-24]. It is well known that systemic proinflammatory cytokines could enter CNS by invading in the impaired blood brain barrier (BBB), accelerating the following occurrence of neurodegeneration [25]. Hence, pro-inflammatory cytokines TNF- $\alpha$, IL- 6 and IL- $1 \beta$ in hippocampus were measured to evaluate the severity of inflammation injury in the brain of AD rats [26]. The acquired data suggested that EX might attenuate the defect of memory and learning capability through the inhibition of pro-inflammatory cytokines.

Three major upstream mechanisms related NLRP3 activation have been proposed: reactive oxygen species (ROS), ion fluxes, or phagosomal destabilization [27]. Therefore, we focused on the ROS, the origin of oxidative stress by examination the generation of oxidative stress markers (MDA, SOD, CAT and GSH-Px) [28]. Varying degree of decrease in the MDA content were observed in the serum in $A \beta_{1-42}$-induced rats treated with EX $(2 \mathrm{~g} / \mathrm{kg}$, $6 \mathrm{~g} / \mathrm{kg}$ ), compared with those in model mice stimulated by $\mathrm{A} \beta_{1-42}$. We also examined the effects of EX on the enzymatic activities of intracellular antioxidant enzymes such as SOD, CAT and GSH-Px in the serum. Increased SOD, CAT and GSH-Px activities inhibited by $\mathrm{A} \beta_{1-42}$ injection was observed in the AD rats treated with EX $(2 \mathrm{~g} / \mathrm{kg}, 6 \mathrm{~g} / \mathrm{kg})$. Our results above implied that the antioxidative ability of EX might contribute to alleviate the development of AD.

Previous investigators revealed that the degradation of extracellular $A \beta$ fibrils by microglia is dependent on autophagic processes [17]. Autophagy is also important for the regulation of $\mathrm{A} \beta$-mediated NLRP3 inflammasome activation, thereby affecting neuronal

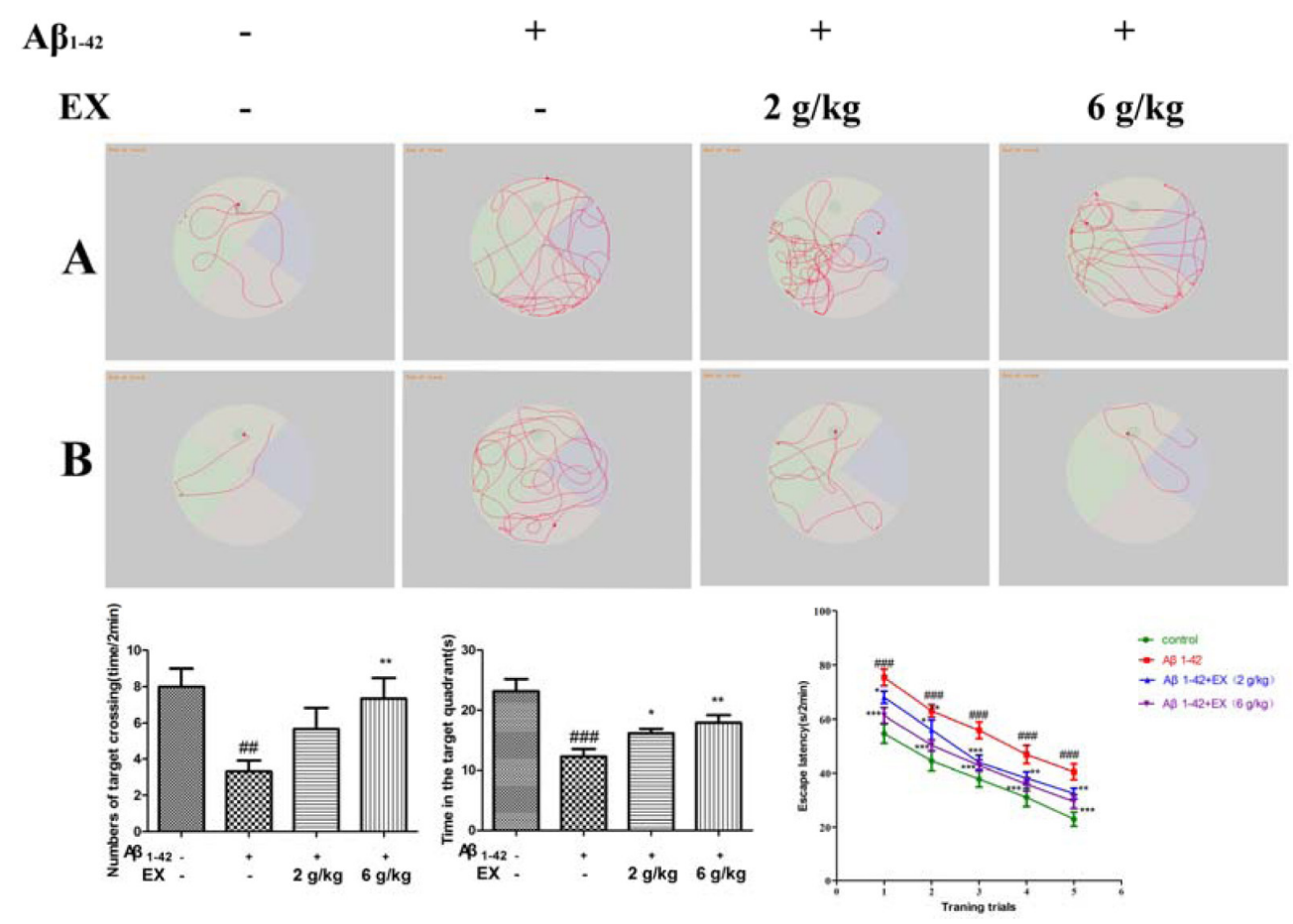

Figure 2: The effect of EX on the performance in Morris water maze of $\mathbf{A} \boldsymbol{\beta}_{1-42}$-induced rats. A. Representative searching strategy of rats in the second trial on the second day. B. Representative searching strategy of rats in the second trial on the fifth day. C. Numbers of target crossing of the rats in all groups. D. The time of the rats spent in the quadrant where the platform was once placed within 120 s. E. Escape latency to find the hidden platform during the five consecutive days training. Data are expressed as mean \pm S.E.M., $n=8$. ${ }^{\#} p<0.05$ versus the control; ${ }^{*} p<0.05$ versus the $\mathrm{A} \beta_{1-42}$-treated group. Data were analyzed with ANOVA followed by Duncan's Multiple Range Test. 
survival. The extracellular accumulation of $\beta$-amyloid $(A \beta)$, regarded as the primary causative factor resulting in neurodegeneration, was thought to be one of the irrefutable pathological findings of AD [29]. Sufficient studies points toward a dysregulation of the immune system and inflammation as potential switch for onset AD. Activated microglia in senile plaques has pro-inflammatory phenotype linked with neurotoxicity [30]. Moreover, A $\beta$ fibrils activate the NLRP3 inflammasome resulting in release of pro-inflammatory cytokine IL-1 $\beta$ in microglia and NLRP3 inflammasome is activated in the brains of AD patients [31]. The results of western blot revealed that the high expressions of NLRP3/ASC/IL-1 $\beta$ pathwayrelated proteins in AD rats were effectively inhibited by EX $(2 \mathrm{~g} / \mathrm{kg}, 6 \mathrm{~g} / \mathrm{kg})$ to different degrees, indicating the impact of NLRP3 on AD and the effectiveness of EX in the treatment of $A D$ rats induced by $A \beta_{1-42}$ administration.

To further identify the molecular mechanism related to the recovery ability of the brain of $\mathrm{AD}$ rats by EX treatment, we utilized the western blot to clarify the relationship between the relative expressions of MAPK and NF- $\kappa$ B pathway in AD rats. Former literature proposed the involvement of MAPK and NF- $\kappa$ B signaling in MAPK and NF- $\mathrm{BB}$ [32]. The closely relationship between MAPK and NF- $\mathrm{BB}$ has been documented [33]. A growing number of links have put forward a strong correlation between MAPK signaling pathway and AD.
$\mathrm{A} \beta$ increased the activation of $\mathrm{JNK}$, which is involved in the following injury and death [34]. Mohammadi et al. indicated JNK inhibitor performed protection against $\mathrm{A} \beta$ through the declined of autophagy and then alleviated memory deficit stimulated by $\mathrm{A} \beta$ [35].Meantime, it is well documented that $\mathrm{NF}-\kappa \mathrm{B}$ is a major regulator for the generation of inflammatory cytokines [36] Upon inflammation stimulation, $\mathrm{I} \kappa \mathrm{B} \alpha$ is phosphorylated at serine residues and degraded rapidly following IKK complex activation [37]. The phosphorylation of p65 subunit is also promoted, and then p65 phosphorylation enhances transcriptional activity of NF- $\kappa \mathrm{B}$ followed by the regulation of pro-inflammatory cytokine production [38]. The data of our study was consistent with previous studies, suggesting that $\mathrm{EX}$ administration lowered $\mathrm{A} \beta_{1-42}$-induced the expression of MAPK related protein and suppressed MAPK-mediated inflammatory response as evidenced by diminished levels of NF- $\kappa$ B p 65 , TNF- $\alpha$ and IL- $1 \beta$ in the hippocampus.

Taken together, our finding described the pharmacological effect of EX on AD. Both inflammation and oxidative stress were considered as the vital factors affecting the function of brain. Besides, the involvement of NLRP3 inflammasome activation might lead to new insights into the underlying pathophysiology of cognition impairment.
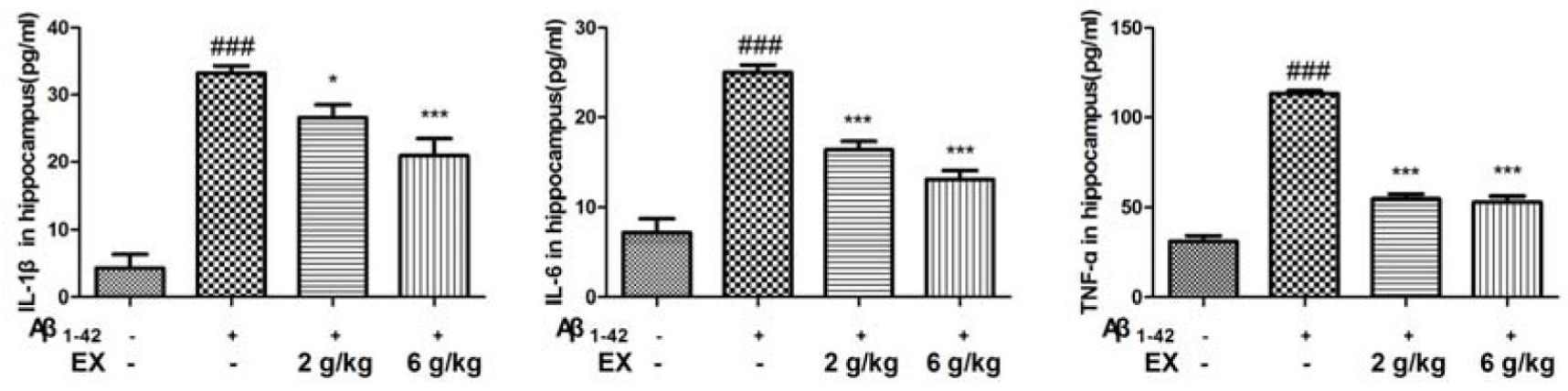

Figure 3: Effects of EX on pro-inflammatory cytokines (IL-1 $\beta$, IL-6 and TNF- $\alpha$ ) in hippocampus of A $\beta_{1-42}$-induced rats. Data were expressed as mean \pm S.E.M. $(n=8)$. ${ }^{\# \#} p<0.01$ and ${ }^{\# \#} p<0.001$ versus the control; ${ }^{*} p<0.05,{ }^{* *} p<0.01$ and ${ }^{* * * *} p<0.001$ versus the $\mathrm{A} \beta_{1-42}$-treated group. Data were analyzed with ANOVA followed by Duncan's Multiple Range Test.
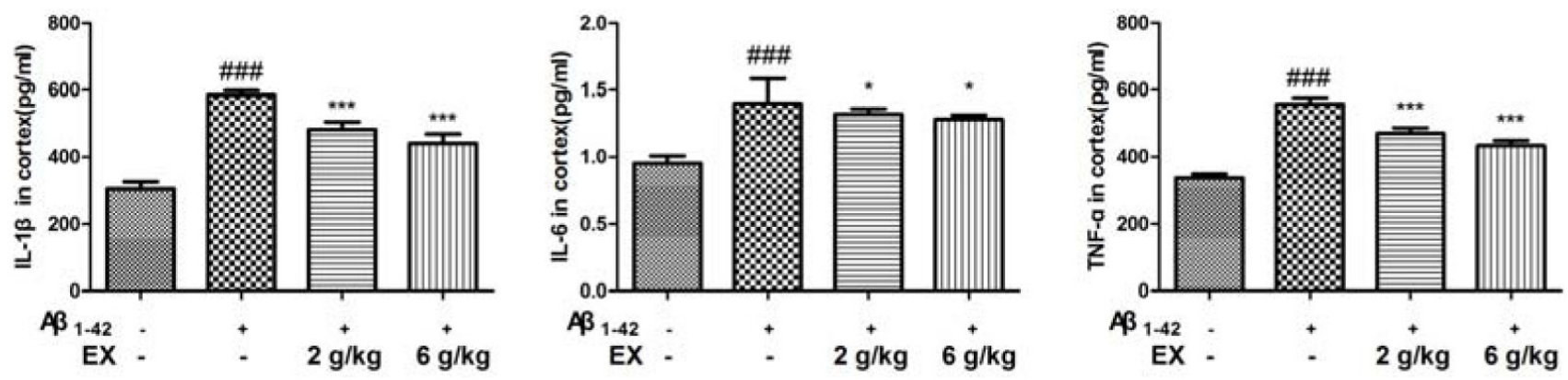

Figure 4: Effects of EX on pro-inflammatory cytokines (IL-1 $\beta$, IL-6 and TNF- $\alpha$ ) in cortex of A $\boldsymbol{\beta}_{1-42}$-induced rats. Data were expressed as mean \pm S.E.M. $(n=8)$. ${ }^{\# \#} p<0.01$ and ${ }^{\# \#} p<0.001$ versus the control; ${ }^{*} p<0.05,{ }^{* *} p<0.01$ and ${ }^{* * *} p<0.001$ versus the $\mathrm{A} \beta_{1-42}$-treated group. Data were analyzed with ANOVA followed by Duncan's Multiple Range Test. 

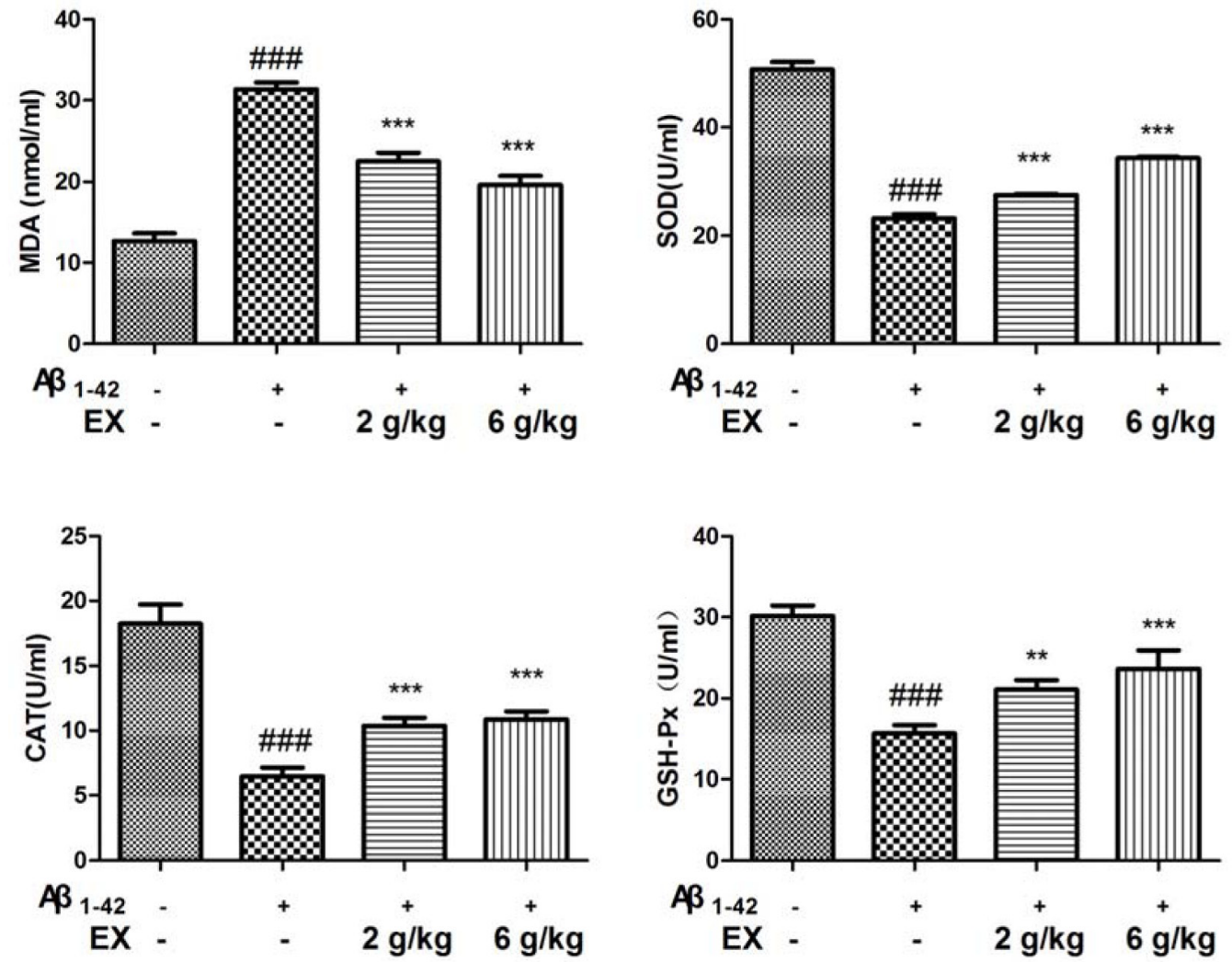

Figure 5: Effects of EX on the levels of SOD, MDA, CAT, GSH-Px in serum of A $\boldsymbol{\beta}_{1-42}$-induced rats. The data expressed as

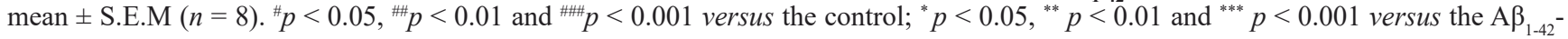
treated group.

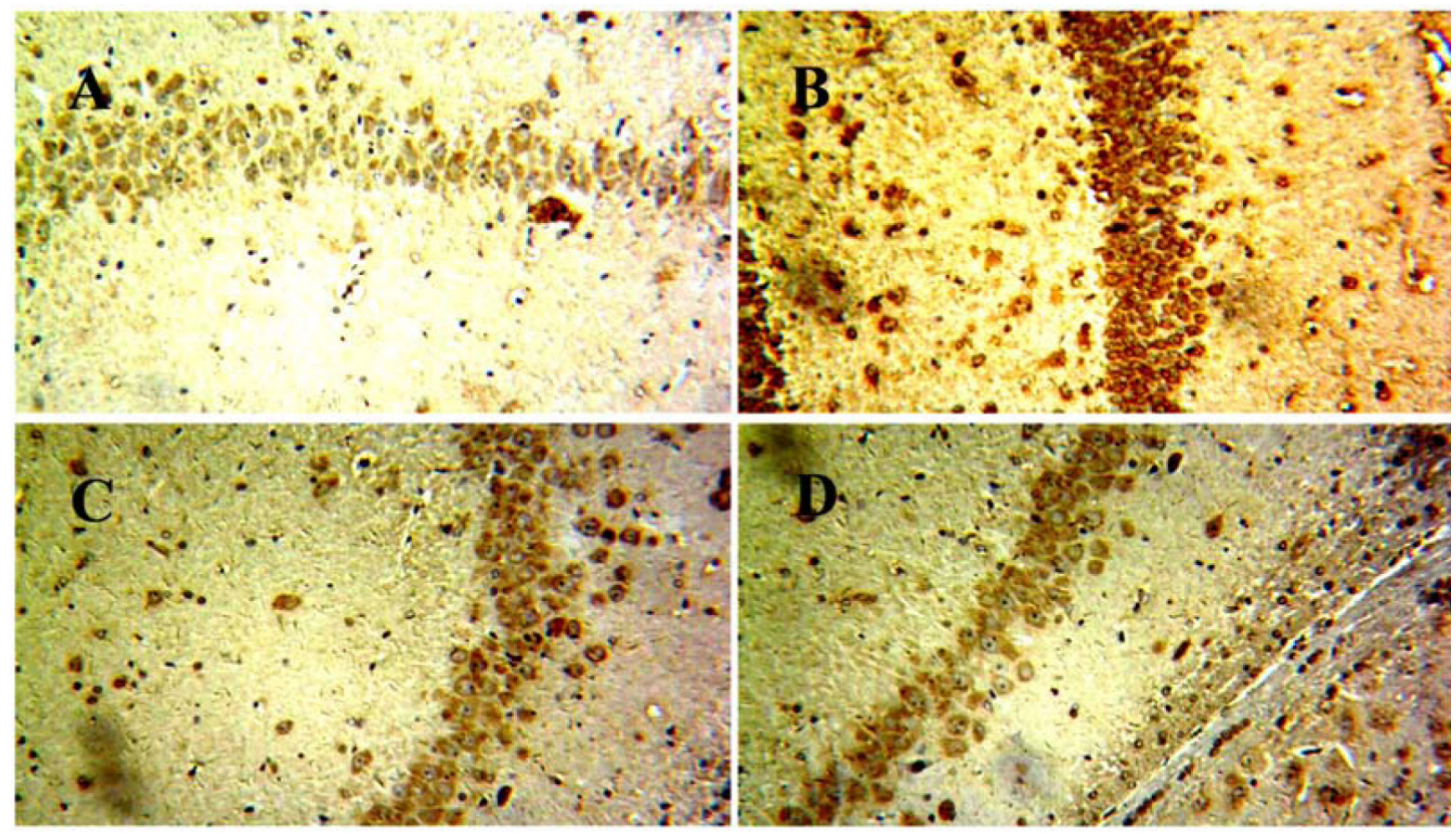

Figure 6: The effects of EX on the expression of NLRP3 in brain of $A \beta_{1-42}$-induced rats by immunohistochemical analysis. A. Contol; B. Model; C. EX(2g/kg); D. EX $(6 \mathrm{~g} / \mathrm{kg})$. 


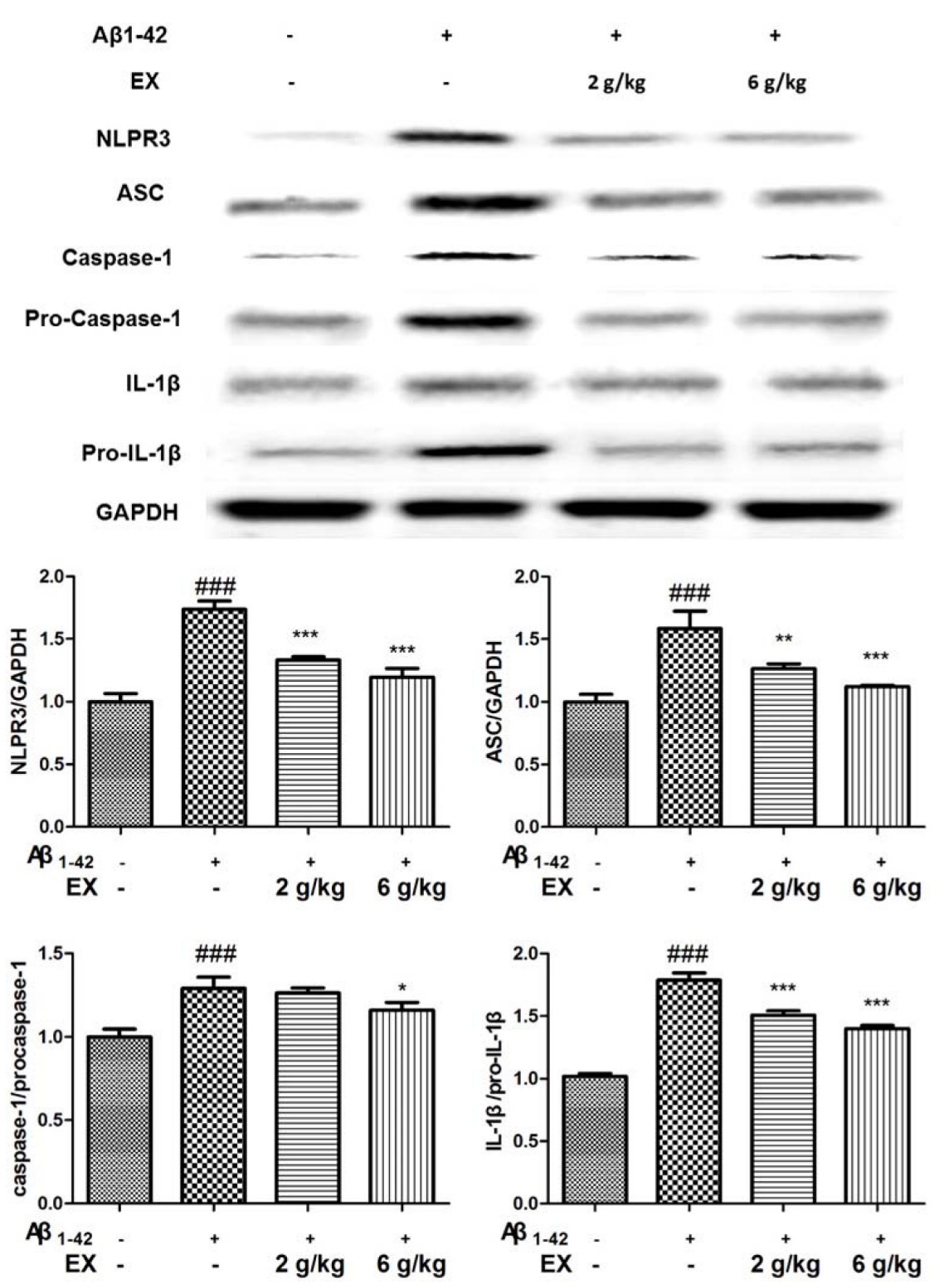

Figure 7: Effects of EX on the activation of NLRP3 inflammasome-related protein of $\mathbf{A}_{1-42}$-induced rats. The data expressed as mean \pm S.E.M $(n=3) .{ }^{\#} p<0.05,{ }^{\# \#} p<0.01$ and ${ }^{\# \#} p<0.001$ versus the control; ${ }^{*} p<0.05,{ }^{* *} p<0.01$ and ${ }^{* * *} p<0.001$ versus the $\mathrm{A} \beta_{1-42}$-treated group.
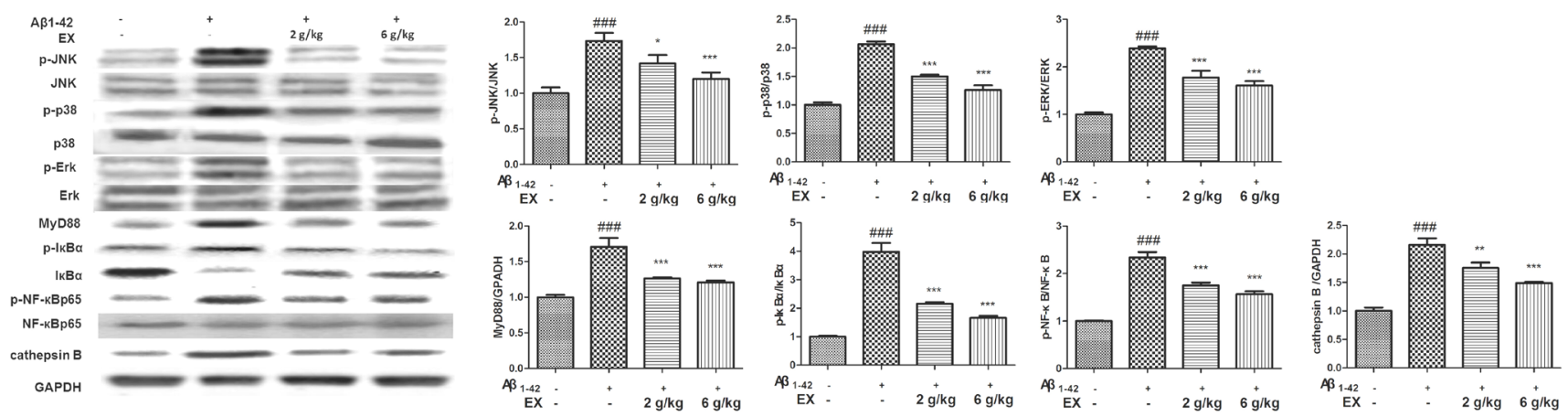

Figure 8: Effects of EX on the activation of MAPK/NF- $\mathrm{KB}$ pathway-related protein of $A \beta_{1-42}$-induced rats. The data expressed as mean \pm S.E.M $(n=3) .{ }^{\#} p<0.05,{ }^{\# p} p<0.01$ and ${ }^{\# \#} p<0.001$ versus the control; ${ }^{*} p<0.05,{ }^{* *} p<0.01$ and ${ }^{* * *} p<0.001$ versus the $\mathrm{A} \beta_{1-42}$-treated group. 


\section{MATERIALS AND METHODS}

\section{Animals}

Adult male Sprague-Dawley rats $(250 \sim 300 \mathrm{~g}$, Comparative Medical Center of Yangzhou University, Yangzhou, China) were housed one week to adapt to their environment before used for experiments. They were maintained on standard laboratory conditions of temperature $25 \pm 1{ }^{\circ} \mathrm{C}$ and a 12-h light/12-h dark cycle with food and water available ad libitum for the duration of the study. All the experiments and animal care were performed strictly in accordance with the Provision and General Recommendation of Chinese Experimental Animals Administration Legislation and were approved by the Science and Technology Department of Jiangsu Province.

\section{Materials and chemicals}

Epimedii Folium and Curculiginis Rhizoma were purchased from Tongrentang drugstore in Wuhan, Hubei province (China). They were authenticated by the corresponding author. The voucher specimens were deposited in the Department of Pharmacognosy, Hubei University of Chinese Medicine. Orcinol glucosid, Curculigoside, Epimedin A, Epimedin B, Icariin and Baohuoside I were purchased from the National Institute for the Control of Pharmaceutical and Biological Products (Beijing, China). The purity of each compound was determined to be higher than $95 \%$ by HPLC. Each reference compound was accurately weighed and dissolved in methanol as stock solutions. Kits used for IL-1 $\beta$, IL-6 and tumor necrosis factor- $\alpha$ (TNF- $\alpha$ ) enzyme linked immunosorbent assay (ELISA) were purchased from Shanghai Westang Bio-tech CO., Ltd., Shanghai, China. Kits for superoxide dismutase (SOD), melonydialdehyde (MDA), and catalase (CAT) were purchased from Nanjing Jiancheng Institute of Biological Engineering (Nanjing, China). Other reagents were AR grade.

Epimedii Folium and Curculiginis Rhizoma were mixed in a ratio of $1: 1$. The mixture was extracted twice for $2 \mathrm{~h}$ each time by distilled water $(1: 10, \mathrm{w} / \mathrm{v})$. The filtrates were concentrated and dried in vacuum at $60^{\circ} \mathrm{C}$, and then stored at $4{ }^{\circ} \mathrm{C}$. The yield of dried powder was $15.5 \%$ according to the original dry material. The doses of water extracts of Epimedii Folium and Curculiginis Rhizoma (EX) were expressed as gram of the original dry materials per kilogram body weight.

\section{TOF-MS analysis of EX}

The multi-components of EX were characterized by TOF-MS. The sample was analyzed using Thermo
Syncronic C18 column $(100 \mathrm{~mm} \times 2.1 \mathrm{~mm}, 1.7 \mu \mathrm{m})$ with the detector wavelength set at $254 \mathrm{~nm}$. The mobile phase consisted of $\mathrm{CH}_{3} \mathrm{CN}(\mathrm{A})$ and $0.1 \%(\mathrm{v} / \mathrm{v}) \mathrm{CH}_{3} \mathrm{COOH}(\mathrm{B})$. A gradient program was used as follows: $0-3 \mathrm{~min}, 5 \%$ A- $95 \%$ B; 3-9 min, 30\% A-70\% B; 9-12 min, $65 \%$ A-35\% B; $12-14 \min , 95 \%$ A-5\% B; 14-15 min, $5 \%$ A- $95 \%$ B. The flow rate was $0.4 \mathrm{~mL} / \mathrm{min}$.

\section{Animal treatment paradigm}

The soluble $\mathrm{A} \beta_{1-42}$ (Sigma-Aldrich, St. Louis, MO, USA) was dissolved in filtered phosphate buffered saline (PBS, pH 7.5, 0.1M) at a concentration of $200 \mu \mathrm{g} / \mu \mathrm{L}$, and then incubated at $37{ }^{\circ} \mathrm{C}$ for seven days. On the test day, the solution was diluted with PBS to reach the final concentration of $1 \mu \mathrm{g} / \mu \mathrm{L}$.

Rats were anaesthetized by intraperitoneal injection of chloral hydrate $(320 \mathrm{mg} / \mathrm{kg})$, and placed into stereotaxic device. After cleaning the area surrounding bregma, $5 \mu \mathrm{L}$ aggregated $\mathrm{A} \beta_{1-42}(1 \mu \mathrm{g} / \mu \mathrm{L})$ or PBS were stereotaxically injected bilaterally into the dorsal hippocampus at a rate of $0.5 \mu \mathrm{L} / \mathrm{min}$. The injected position was coordinated relative to the bregma: $-3.8 \mathrm{~mm}$ posterior to bregma, \pm $2.5 \mathrm{~mm}$ lateral to midline and $-3.0 \mathrm{~mm}$ to the dura ${ }^{39}$. The rats were left in a temperature-controlled chamber until they recovered from anesthesia. Penicillin-G 200,000 $\mathrm{IU} / \mathrm{mL}$ was administered after surgery $(0.2 \sim 0.3 \mathrm{~mL} / \mathrm{rat}$, intramuscular).

One day after the operation, the $\mathrm{A} \beta_{1-42}$-treated rats were randomly divided into 3 groups as follows: group $2(n=8)$ that served as model group received the PBS (p.o.); group $3(n=8)$ and $4(n=8)$ received $\mathrm{EX}$ at the doses of $2 \mathrm{~g} / \mathrm{kg}$ and $6 \mathrm{~g} / \mathrm{kg}$ (p.o.), respectively. Meanwhile, PBS-intrahippocampal injected rats served as control group $(n=8)$ received the PBS (p.o.). The drugs were orally administered through feeding tube daily for 30 consecutive days.

\section{Morris water maze test}

Spatial memory ability was detected by Morris water maze test [40] with minor modifications. The Morris water maze, which consisted of 5-6 days place navigation training and a probe test on the day 6 , was carried out in a black circular pool $(180 \mathrm{~cm}$ in diameter and $60 \mathrm{~cm}$ in height) with a featureless inner surface. A round escape platform was placed in $1 \mathrm{~cm}$ underneath the water surface in the center of one quadrant. The rats were given two trial sessions each day for five consecutive days, with an inter-trial interval of $20 \mathrm{~min}$, and the escape latencies were recorded. Once the rat located the platform, it was permitted to remain on it for $10 \mathrm{~s}$. If the rat did not locate the platform within $120 \mathrm{~s}$, it was placed on the platform for $15 \mathrm{~s}$ and the escape latency was recorded as $120 \mathrm{~s}$. On day 6 , the platform was removed and each rat was allowed 
to swim freely for $120 \mathrm{~s}$ as the probe test. The time that rat spent in the target quadrant (where the platform was once hidden) was measured.

\section{Preparation of tissue samples}

Rats were decapitated 60 min after the behavioral tests. Brains were removed carefully and quickly to $0.9 \%$ cold saline and the hippocampus were immediately dissected on a cold plate, weighed and homogenized with ice-cold saline. The homogenate was centrifuged at 3000 rpm for $10 \mathrm{~min}$ at $4{ }^{\circ} \mathrm{C}$ and the supernatant was used.

\section{Determination of cytokines (IL-1 $\beta$, IL-6, TNF- $\alpha$, MDA, SOD, CAT and GSH-Px)}

Levels of IL-1 $\beta$, IL- 6 and TNF- $\alpha$ in hippocampus and cortex were determined by ELISA kits according to the manufacture instructions. Cytokine concentrations in the test samples were evaluated with reference to the standard curves prepared using recombinant cytokines of known concentrations.

Activities of MDA, SOD, CAT and GSH-Px in the serum were also measured by the methods described in the assay kits. SOD activities $(\mathrm{U} / \mathrm{ml})$ of samples were expressed with reference to the activity of a standard curve of bovine erythrocyte SOD dilutions. One unit was defined as the amount of enzyme needed to exhibit fifty percent dismutation of the superoxide radical. Absorbance values of both samples and standards were measured at $440 \mathrm{~nm}$ by a micro-plate reader. The amounts of TBARS such as MDA $(\mathrm{nmol} / \mathrm{ml})$ in samples were measured by the reaction with TBA. CAT activities $(\mathrm{U} / \mathrm{ml})$ of samples were measured on the basis of the activity that catalyzed the conversion of $\mathrm{H} 2 \mathrm{O} 2$ to oxygen and water. GSH-Px activities $(\mathrm{U} / \mathrm{ml})$ of samples were measured by the content of NADPH.

\section{Western blot analysis}

The hippocampus was lysed in RIPA buffer containing protease or phosphatase inhibitors. Protein concentrations were determined by the Bradford protein assay. Protein lysates were separated by SDS-PAGE electrophoresis and were transferred onto polyvinylidene difluoride (PVDF) membranes. Antibodies for Western blots were from Cell Signaling Technology unless indicated. Products numbers and antibody dilutions are indicated within parentheses. After blocking with 5\% BSA for 1 hour, the membranes were incubated at $4{ }^{\circ} \mathrm{C}$ overnight with the following antibodies: NLPR3 (\#15101, 1:1000), ASC (\#13833, 1:1000), caspase-1 (\#2225, 1:1000), pro-caspase-1 (\#2225, 1:1000), IL-1 $\beta$ (\#12703, 1:1000), pro-IL-1 $\beta$ (\#12703, 1:1000), NF-кB p65 (\#3034,
1:1000), phospho-NF-кB p65 (\#3031, 1:1000), JNK (\#4672, 1:1000), p-JNK (\#4668, 1:1000), P38 (\#8690, 1:1000), p-P38 (\#4511, 1:1000), Erk (\#4695, 1:1000), p-Erk (\#4094, 1:1000), IкB $\alpha(\# 4766,1: 1000), \mathrm{p}-\mathrm{I} \kappa \mathrm{B} \alpha$ (\#4766, 1:1000), MyD88 (\#4283, 1:1000), cathepsin B (Santa Cruz Biotechnology, sc-13985, 1:500), GAPDH (\#5174, 1:1000). Subsequently, the membranes were incubated with the appropriate horseradish peroxidaseconjugated secondary antibody for 1 hour. Bands intensity was measured using the SuperSignal West Pico Chemiluminescent Substrate (Thermo Fisher Scientific Inc.) and normalized by corresponding loading control proteins.

\section{Statistical analysis}

All values were expressed as the mean \pm S.E.M. and analyzed by one-way analysis of variance (ANOVA) followed by Duncan's Multiple Range Test using SPSS version 13.0 software; a $p$-value of less than 0.05 was considered significant.

\section{Abbreviations}

A $\beta, \beta$-amyloid peptide; AD, Alzheimer's disease; CAT, catalase; EX, the water extracts of Epimedii Folium and Curculiginis Rhizoma; IL-1 $\beta$, interleukin-1 $\beta$; MDA, melonydialdehyde; NF- $\kappa \mathrm{B}$, nuclear factor $\kappa \mathrm{B}$; SOD, superoxide dismutase; TNF- $\alpha$, tumor necrosis factor- $\alpha$.

\section{ACKNOWLEDGMENTS}

This work was supported by the National Natural Science Foundation of China (81303284, 81403157 and 81130064), the "Chenguang Planning" of the Natural Science Foundation of Wuhan City (2015070404010183), and China Postdoctoral Science Foundation (2015M571243 and 2016T90193).

\section{CONFLICTS OF INTEREST}

The authors declare that they have no conflicts of interest to disclose.

\section{REFERENCES}

1. Khan MZ, Zhuang X, He L. GPR40 receptor activation leads to CREB phosphorylation and improves cognitive performance in an Alzheimer's disease mouse model. Neurobiol Learn Mem. 2016; 131:46-55.

2. Chen Y, Yang X, Chen T, Ji J, Lan L, Hu R, Ji H. Treatment with Akebia Saponin D Ameliorates A $\beta 1-42-$ Induced Memory Impairment and Neurotoxicity in Rats. Molecules. 2016; $21: 323$. 
3. Prince M, Bryce R, Ferri C. World Alzheimer Report 2011: The benefits of early diagnosis and intervention. Alzheimer's Disease International; 2011.

4. Martin BC, Ricci JF, Kotzan JA, Lang K, Menzin J. The net cost of Alzheimer disease and related dementia: a population-based study of Georgia Medicaid recipients. Alzheimer Dis Assoc Disord. 2000; 14:151-59.

5. Spangenberg EE, Lee RJ, Najafi AR, Rice RA, Elmore MR, Blurton-Jones M, West BL, Green KN. Eliminating microglia in Alzheimer's mice prevents neuronal loss without modulating amyloid- $\beta$ pathology. Brain. 2016; 139:1265-81.

6. Chen T, Ma Z, Zhu L, Jiang W, Wei T, Zhou R, Luo F, Zhang K, Fu Q, Ma C, Yan T. Mol Neurobiol. 2015.

7. Licastro F, Pedrini S, Caputo L, Annoni G, Davis LJ, Ferri C, Casadei V, Grimaldi LM. Increased plasma levels of interleukin-1, interleukin-6 and alpha-1-antichymotrypsin in patients with Alzheimer's disease: peripheral inflammation or signals from the brain? J Neuroimmunol. 2000; 103:97102.

8. Jiang Q, Yi M, Guo Q, Wang C, Wang H, Meng S, Liu $\mathrm{C}, \mathrm{Fu}$ Y, Ji H, Chen T. Protective effects of polydatin on lipopolysaccharide-induced acute lung injury through TLR4-MyD88-NF-кB pathway. Int Immunopharmacol. 2015; 29:370-76.

9. Zhang K, Liu J, You X, Kong P, Song Y, Cao L, Yang S, Wang W, Fu Q, Ma Z. P2X7 as a new target for chrysophanol to treat lipopolysaccharide-induced depression in mice. Neurosci Lett. 2016; 613:60-65.

10. Tan MS, Yu JT, Jiang T, Zhu XC, Tan L. The NLRP3 inflammasome in Alzheimer's disease. Mol Neurobiol. 2013; 48:875-82.

11. Tayeb HO, Yang HD, Price BH, Tarazi FI. Pharmacotherapies for Alzheimer's disease: beyond cholinesterase inhibitors. Pharmacol Ther. 2012; 134:8-25.

12. $\mathrm{Wu} \mathrm{X}, \mathrm{Wu}$ J, Xia S, Li B, Dong J. Icaritin opposes the development of social aversion after defeat stress via increases of GR mRNA and BDNF mRNA in mice. Behav Brain Res. 2013; 256:602-08.

13. Cui Z, Sheng Z, Yan X, Cao Z, Tang K. In Silico Insight into Potential Anti-Alzheimer's Disease Mechanisms of Icariin. Int J Mol Sci. 2016; 17:113.

14. Chauhan NS, Rao CV, Dixit VK. Effect of Curculigo orchioides rhizomes on sexual behaviour of male rats. Fitoterapia. 2007; 78:530-34.

15. Lakshmi V, Pandey K, Puri A, Saxena RP, Saxena KC. Immunostimulant principles from Curculigo orchioides. J Ethnopharmacol. 2003; 89:181-84.

16. Qin L, Han T, Zhang Q, Cao D, Nian H, Rahman K, Zheng H. Antiosteoporotic chemical constituents from ErXian Decoction, a traditional Chinese herbal formula. J Ethnopharmacol. 2008; 118:271-79.

17. Cho MH, Cho K, Kang HJ, Jeon EY, Kim HS, Kwon HJ, Kim HM, Kim DH, Yoon SY. Autophagy in microglia degrades extracellular $\beta$-amyloid fibrils and regulates the NLRP3 inflammasome. Autophagy. 2014; 10:1761-75.

18. Khan MZ, He L. Neuropharmacology. 2017;113:639-651.

19. Gao J, He H, Jiang W, Chang X, Zhu L, Luo F, Zhou R, Ma C, Yan T. Salidroside ameliorates cognitive impairment in a d-galactose-induced rat model of Alzheimer's disease. Behav Brain Res. 2015; 293:27-33.

20. Yu X, Wang LN, Ma L, You R, Cui R, Ji D, Wu Y, Zhang $\mathrm{CF}$, Yang ZL, Ji H. Akebia saponin D attenuates ibotenic acid-induced cognitive deficits and pro-apoptotic response in rats: involvement of MAPK signal pathway. Pharmacol Biochem Behav. 2012; 101:479-86.

21. Yin Z, Yu H, Chen S, Ma C, Ma X, Xu L, Ma Z, Qu R, Ma S. Asiaticoside attenuates diabetes-induced cognition deficits by regulating PI3K/Akt/NF- $\mathrm{KB}$ pathway. Behav Brain Res. 2015; 292:288-99.

22. Zhong SZ, Ge QH, Li Q, Qu R, Ma SP. Peoniflorin attentuates $\operatorname{Abeta}((1-42))$-mediated neurotoxicity by regulating calcium homeostasis and ameliorating oxidative stress in hippocampus of rats. J Neurol Sci. 2009; 280:7178.

23. Zhu L, Chen T, Chang X, Zhou R, Luo F, Liu J, Zhang K, Wang Y, Yang Y, Long H, Liu Y, Yan T, Ma C. Salidroside ameliorates arthritis-induced brain cognition deficits by regulating $\mathrm{Rho} / \mathrm{ROCK} / \mathrm{NF}-\kappa \mathrm{B}$ pathway. Neuropharmacology. 2016; 103:134-42.

24. Chen T, Guo Q, Wang H, Zhang H, Wang C, Zhang P, Meng S, Li Y, Ji H, Yan T. Effects of esculetin on lipopolysaccharide (LPS)-induced acute lung injury via regulation of RhoA/Rho Kinase/NF-кB pathways in vivo and in vitro. Free Radic Res. 2015; 49:1459-68.

25. Jiang W, Zhou R, Li P, Sun Y, Lu Q, Qiu Y, Wang J, Liu J, Hao K, Ding X. Protective effect of chrysophanol on LPS/ $\mathrm{d}$-GalN-induced hepatic injury through the RIP140/NF- $\mathrm{BB}$ pathway. RSC Advances. 2016; 6:38192-200.

26. Gao J, Zhou R, You X, Luo F, He H, Chang X, Zhu L, Ding X, Yan T. Salidroside suppresses inflammation in a D-galactose-induced rat model of Alzheimer's disease via SIRT1/NF-кB pathway. Metab Brain Dis. 2016; 31:771-78.

27. Xiang P, Chen T, Mou Y, Wu H, Xie P, Lu G, Gong X, Hu Q, Zhang Y, Ji H. NZ suppresses TLR4/NF- $\mathrm{B}$ signalings and NLRP3 inflammasome activation in LPS-induced RAW264.7 macrophages. Inflamm Res. 2015;64:799-808.

28. Chen T, Jiang W, Zhang H, You X, Liu M, Wang L, Xiang P, Xu L, Zheng D, Zhang X, Ji H, Hao K, Yan T. Protective effect of trillin against ethanol-induced acute gastric lesions in an animal model. RSC Advances. 2016; 6:20081-88.

29. Wang SY, Liu JP, Ji WW, Chen WJ, Fu Q, Feng L, Ma SP. Qifu-Yin attenuates AGEs-induced Alzheimer-like pathophysiological changes through the RAGE/NF- $\mathrm{BB}$ pathway. Chin J Nat Med. 2014; 12:920-28.

30. Halle A, Hornung V, Petzold GC, Stewart CR, Monks BG, Reinheckel T, Fitzgerald KA, Latz E, Moore KJ, Golenbock DT. The NALP3 inflammasome is involved in the innate 
immune response to amyloid-beta. Nat Immunol. 2008; 9:857-65.

31. Heneka MT, Kummer MP, Stutz A, Delekate A, Schwartz S, Vieira-Saecker A, Griep A, Axt D, Remus A, Tzeng TC, Gelpi E, Halle A, Korte M, et al. NLRP3 is activated in Alzheimer's disease and contributes to pathology in APP/ PS1 mice. Nature. 2013; 493:674-78.

32. Yu X, Wang LN, Du QM, Ma L, Chen L, You R, Liu L, Ling JJ, Yang ZL, Ji H. Akebia Saponin D attenuates amyloid $\beta$-induced cognitive deficits and inflammatory response in rats: involvement of $\mathrm{Akt} / \mathrm{NF}-\mathrm{\kappa B}$ pathway. Behav Brain Res. 2012; 235:200-09.

33. Chen T, Mou Y, Tan J, Wei L, Qiao Y, Wei T, Xiang P, Peng S, Zhang Y, Huang Z, Ji H. The protective effect of CDDO-Me on lipopolysaccharide-induced acute lung injury in mice. Int Immunopharmacol. 2015; 25:55-64.

34. Bamji-Mirza M, Callaghan D, Najem D, Shen S, Hasim MS, Yang Z, Zhang W. Stimulation of insulin signaling and inhibition of JNK-AP1 activation protect cells from amyloid- $\beta$-induced signaling dysregulation and inflammatory response. J Alzheimers Dis. 2014; 40:105-22.

35. Mohammadi M, Guan J, Khodagholi F, Yans A, Khalaj S, Gholami M, Taghizadeh GH, Aliaghaei A, Abdollahi M, Ghahremani MH, Sharifzadeh M. Reduction of autophagy markers mediated protective effects of JNK inhibitor and bucladesine on memory deficit induced by $A \beta$ in rats. Naunyn Schmiedebergs Arch Pharmacol. 2016; 389:50110.
36. Jiang W, Luo F, Lu Q, Liu J, Li P, Wang X, Fu Y, Hao K, Yan T, Ding X. The protective effect of Trillin LPS-induced acute lung injury by the regulations of inflammation and oxidative state. Chem Biol Interact. 2016; 243:127-34.

37. Chang X, Luo F, Jiang W, Zhu L, Gao J, He H, Wei T, Gong S, Yan T. Protective activity of salidroside against ethanol-induced gastric ulcer via the MAPK/NF- $\kappa \mathrm{B}$ pathway in vivo and in vitro. Int Immunopharmacol. 2015; 28:604-15.

38. Chen T, Wang R, Jiang W, Wang H, Xu A, Lu G, Ren Y, $\mathrm{Xu}$ Y, Song Y, Yong S, Ji H, Ma Z. Protective Effect of Astragaloside IV Against Paraquat-Induced Lung Injury in Mice by Suppressing Rho Signaling. Inflammation. 2016; 39:483-92.

39. Lan Z, Chen L, Fu Q, Ji W, Wang S, Liang Z, Qu R, Kong L, Ma S. Paeoniflorin attenuates amyloid-beta peptideinduced neurotoxicity by ameliorating oxidative stress and regulating the NGF-mediated signaling in rats. Brain Res. 2013; 1498:9-19.

40. Lan Z, Liu J, Chen L, Fu Q, Luo J, Qu R, Kong L, Ma S. Danggui-Shaoyao-San ameliorates cognition deficits and attenuates oxidative stress-related neuronal apoptosis in d-galactose-induced senescent mice. J Ethnopharmacol. 2012; 141:386-95. 